\title{
Optimización del rotor de un alternador inductor heteropolar con inversión de flujo
}

\author{
Fernando Martínez y Javier de las Morenas \\ Escuela de Ingeniería Minera e Industrial, Depto. de Ingeniería Eléctrica, Electrónica, Automática y Comunicaciones, \\ Universidad de Castilla La Mancha. Plaza Manuel Meca 1, 13400 Almadén, (Ciudad Real), España. \\ (correo-e: Fernando.MGarcia@uclm.es; Javier.delasMorenas@uclm.es)
}

Recibido Ago. 29, 2019; Aceptado Oct. 23, 2019; Versión final Dic. 6, 2019, Publicado Abr. 2020

\section{Resumen}

Se utiliza el núcleo del estator de una máquina eléctrica para aplicaciones docentes, con 12 dientes y ranuras abiertas, y se construye un rotor, de material magnético sinterizado, para configurar un alternador inductor heteropolar trifásico, con inversión de flujo. La optimización del diseño del rotor consiste en obtener el mejor número de dientes que proporcione una forma de onda de la tensión de salida más próxima a una función sinusoidal y con un mayor valor eficaz (rms). Se presenta un modelo analítico que permite establecer una comparación de los valores de las permeancias, según sea el número de dientes del rotor. Los resultados se contrastan con valores de simulación, utilizando un programa de elementos finitos. Se concluye que siete es el número idóneo de dientes y ranuras del rotor.

Palabras clave: alternador inductor heteropolar; alternador con inversión de flujo; generador de alta frecuencia, análisis de elementos finitos

\section{Rotor optimization of a flux switching heteropolar inductor alternator}

\begin{abstract}
The stator core of an electric machine for teaching applications, with 12 teeth and open slots, is used and a rotor, of sintered magnetic material, is constructed to configure a flux switching three-phase heteropolar inductor alternator. The optimization of the rotor design consists of obtaining the best number of teeth that provides a waveform of the output voltage closest to a sinusoidal function and with a higher effective value (rms). An analytical model is presented, allowing to establish a comparison of the values of the permeances, depending on the number of teeth of the rotor. Results are compared with simulation values, using a finite element program. The conclusion reached is that seven is the ideal number of teeth and slots of the rotor.
\end{abstract}

Keywords: heteropolar inductor alternator; flux switching alternator; high frequency generator; finite element analysis 


\section{INTRODUCCIÓN}

Los alternadores inductores se desarrollaron desde finales del siglo XIX, formando parte de convertidores electromecánicos para la generación de energía eléctrica en media y alta frecuencia (Lamme, 1904). A lo largo del siglo XX se utilizaron en procesos tecnológicos, como la fusión eléctrica, soldadura, endurecimiento superficial y electrosecado, que requerían fuentes de alimentación con frecuencias comprendidas entre 1.000 y $10.000 \mathrm{~Hz}$ (Marchbanks, 1946). Actualmente encuentran aplicación, en el funcionamiento a altas velocidades de rotación, acoplados al eje de una turbina de gas (Sivasubramaniam et al., 2009), y, también, en vehículos híbridos (Zhang et al., 2016) o en volantes de inercia (Severson et al., 2015) y, a bajas velocidades, en sistemas eólicos de generación de energía eléctrica (Akuru y Kamper, 2018). Pueden encontrarse, realizando una búsqueda en Internet, empresas que incorporan alternadores inductores; por ejemplo, en sistemas de iluminación y aire acondicionado en ferrocarriles (Kerala Electrical Company), o en grandes vehículos especiales para minería y obra civil (Milspec Manufacturing). Generalmente fabrican, por encargo, para instituciones gubernamentales o militares, por lo que la información es muy restringida.

El alternador inductor presenta algunas ventajas sobre las máquinas eléctricas que tienen un devanado en el estator y el otro devanado en el rotor, que hacen que sea muy adecuado para aplicaciones de alta velocidad (Li et al., 2016). Se compone de un rotor sólido simple, que no tiene arrollamientos. El estator lleva dos conjuntos de devanados, excitación e inducido. La tensión se induce por la variación del flujo magnético, $(\phi)$, producido por el devanado de excitación, debido a cambios en la permeancia magnética, a medida que el rotor gira. Existen dos tipos, el alternador inductor homopolar y el heteropolar (Walker, 1942). En los alternadores inductores heteropolares, dependiendo de la posición relativa entre los devanados de excitación y de inducido, el flujo ligado a una bobina del inducido puede ser pulsante o cambiar de sentido. Así, en la configuración de la Figura 1a el flujo es pulsante, oscilando entre los valores máximo y mínimo, mientras que en la configuración de la Figura $1 \mathrm{~b}$ el flujo total ligado a cada una de las bobinas del inducido invierte su sentido al girar el rotor. Se representan en negro las bobinas de excitación, indicando el sentido del flujo que crean, y en azul, verde o rojo las bobinas de cada una de las fases de un inducido trifásico.

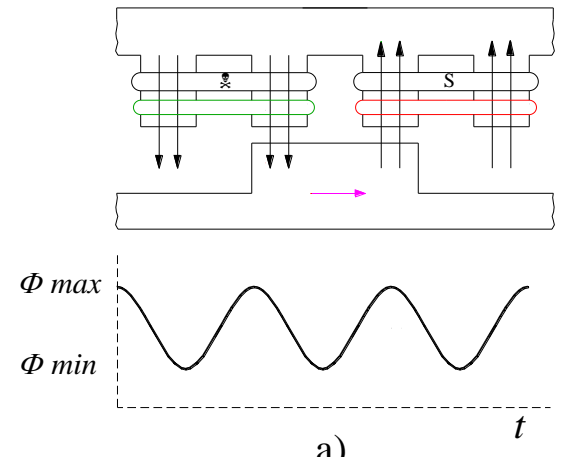

a)

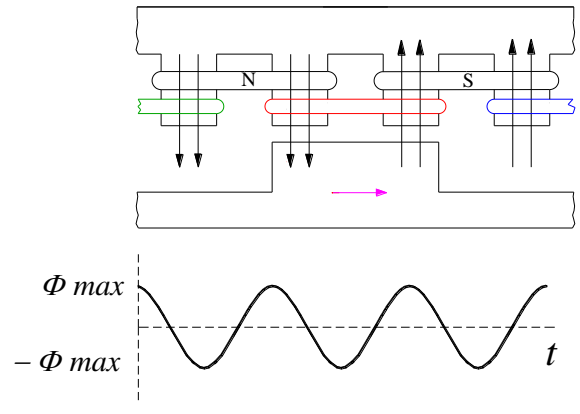

b)

Fig. 1: Alternador inductor heteropolar. a) Flujo pulsante. b) Flujo con inversión de sentido.

Rauch y Johnson (1955) introdujeron el concepto de alternador con inversión del flujo, (flux switching alternator). Se compone, en el caso más sencillo, de un estator laminado con cuatro polos, en el que el devanado de excitación (en negro) y el devanado del inducido (en azul) están dispuestos de forma alternada. El devanado de excitación puede ser sustituido por imanes permanentes. (Figura 2).

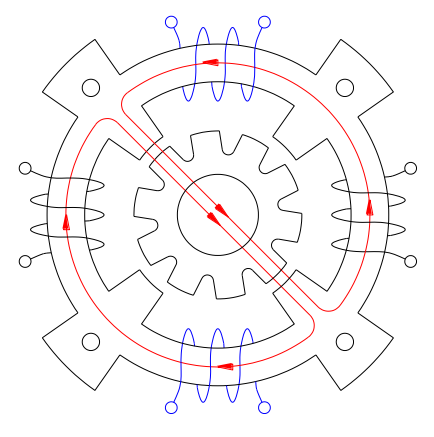

a)

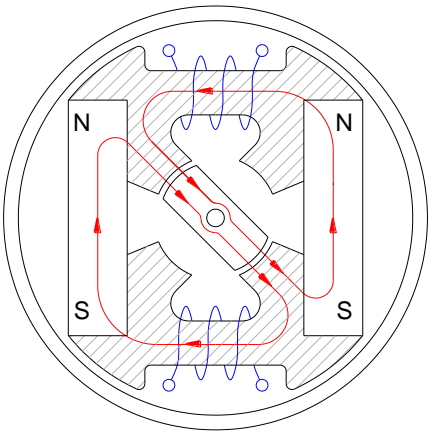

b)

Fig. 2: Configuraciones iniciales del alternador con inversión del flujo. a) Con devanado de excitación de corriente continua. b) Con imanes permanentes. 
Las diferentes topologías actuales de los alternadores inductores heteropolares con inversión del flujo están basadas en criterios que tienen en cuenta el tipo de excitación, (corriente continua, imanes permanentes, híbrida), la orientación del flujo en el entrehierro, (radial o axial) y la construcción del núcleo del estator y del rotor, (forma y número de dientes) (Shen y Fei, 2013). Aunque las configuraciones más utilizadas tienen únicamente excitación mediante imanes permanentes, resulta difícil regular la tensión de salida, puesto que no puede modificarse el valor del campo magnético producido por los imanes. Esto hace que, en algunas aplicaciones, sea aconsejable utilizar un devanado de corriente continua para la excitación, en lugar de imanes permanentes (Zhu et al., 2015). La excitación híbrida combina las ventajas del devanado de corriente continua y de los imanes permanentes y permite un mejor control del campo magnético, aunque aumenta la complejidad constructiva (Gaussens et al., 2014).

\section{MODELO}

La configuración elegida es una modificación del alternador heteropolar del tipo Lorentz, con las ranuras abiertas (Walker, 1946; Das Gupta, 1960). El número de dientes y ranuras del estator es 12. El generador es trifásico y tiene 2 bobinas de inducido, por fase. Las bobinas del inducido pueden configurarse con pasos 1 $3,1-5$ o 1-7. Se elige el paso 1-3, lo que hace que cada bobina abrace a dos dientes del estator. Se disponen 6 bobinas de excitación, conectadas en serie, de tal forma que constituyan alternativamente polos norte (N) y sur (S). La disposición, en las ranuras del estator, de las bobinas de excitación y del inducido es alternada, de forma que cada bobina del inducido está ubicada entre un polo de excitación $\mathrm{N}$ y otro S. (Figura 3).

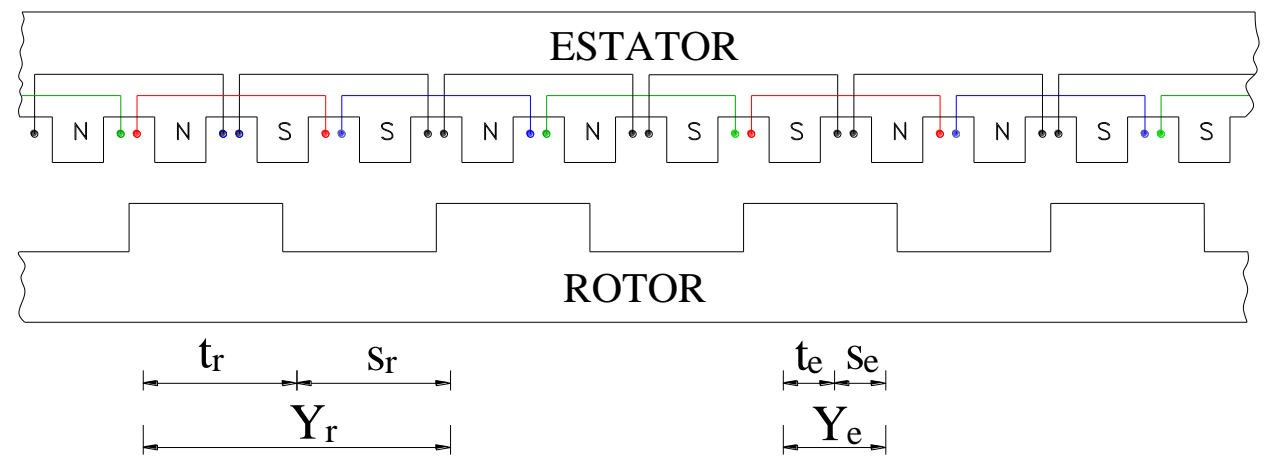

Fig. 3: Desarrollo del alternador inductor trifásico objeto de análisis.

Cuando el rotor gira, con una velocidad angular $\Omega$, la permeancia $(P)$ de las trayectorias del flujo magnético ligado a cada bobina cambia, debido a la variación del tamaño del entrehierro entre los dientes del estator y del rotor. $(P=\mu S / I ; \mu=$ Permeabilidad magnética, $S=$ sección transversal, $I=$ longitud). Los cambios en la permeancia hacen que varíe el flujo ligado a cada bobina, lo que provoca la generación de tensión en las bobinas del inducido. La magnitud y la forma de la onda del flujo dependen del número de dientes del rotor y también de la relación entre los anchos de diente y ranura del rotor. Puede obtenerse el número óptimo de dientes del rotor para el cual el generador proporciona la mejor forma de onda en circuito abierto, en cuanto a su valor eficaz y el mínimo contenido en armónicos.

Es condición fundamental que el devanado de excitación, considerado en su totalidad, presente permeancia constante para cualquier posición del rotor. De no ser así se produciría una variación en el flujo magnético total ligado a este devanado que provocaría la autoinducción de tensión y, por tanto, la aparición de una corriente alternante superpuesta a la corriente continua de la excitación, lo que empeoraría las prestaciones del alternador.

Sin hipótesis simplificativas, el análisis para determinar el número óptimo de dientes del rotor resultaría extremadamente complicado. En consecuencia, se asumirán tres suposiciones: 1. La permeancia de las trayectorias del flujo, en cada diente del estator, varía sinusoidalmente con el ángulo mecánico $\theta$, como consecuencia de la rotación del rotor, $(\theta=\Omega t)$, y, por lo tanto, la variación del flujo en cada diente del estator, (en un arco de longitud igual al paso polar $Y_{e}$ ), es sinusoidal, ya que $\phi=\left(N_{e} l_{e}\right) \cdot P$. (Figura 4. $N_{e} l_{e}$ es la fuerza magnetomotriz del devanado de excitación); 2. La permeancia en los tramos de hierro es infinita en comparación con la de los tramos de aire; y 3. El paso polar del rotor, $\left(Y_{r}\right)$, es mayor o igual que el del estator, $\left(Y_{e}\right)$. El ancho de diente, $\left(t_{e}\right)$, y de ranura del estator, $\left(\mathrm{S}_{\mathrm{e}}\right)$, son iguales entre sí y también son iguales entre sí el ancho de diente, $\left(\mathrm{tr}_{\mathrm{r}}\right)$, y de ranura del rotor, $\left(\mathrm{s}_{\mathrm{r}}\right)$. (Figura 3 ). 


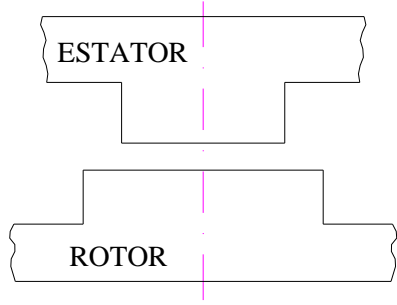

a)

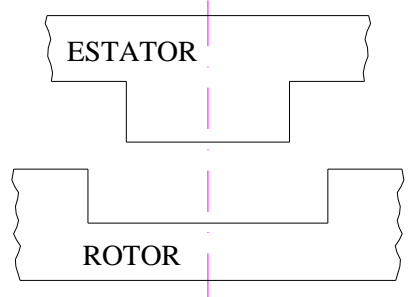

b)

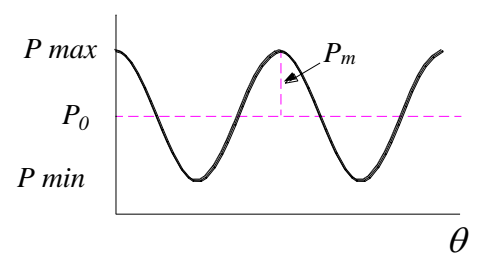

c)

Fig. 4: a) Configuración de máxima permeancia. b) Configuración de mínima permeancia. c) Variación de la permeancia al girar el rotor.

\section{ANÁLISIS}

La expresión de la variación de la permeancia en un diente del estator, (Figura 4), tomando la situación representada en la Figura 4a como instante inicial, es:

$$
P(\theta)=P_{0}+P_{m} \cos \theta
$$

$P_{0}=$ Valor medio; $P_{m}=$ Amplitud de la onda sinusoidal $\theta=$ Ángulo girado por el rotor .

Puesto que cada bobina de fase del inducido abraza a dos dientes del estator, uno de los cuales es un polo $\mathrm{N}$ y el otro un polo $\mathrm{S}$, el flujo total efectivo, a través de cada bobina de fase, como consecuencia de la corriente continua que circula por el devanado de excitación, será el resultante debido a los polos $\mathrm{N}$ y S abrazados por ella. $\mathrm{Si}$ el flujo debido al polo $\mathrm{N}$ se considera positivo, el flujo debido al polo $\mathrm{S}$ debe considerarse negativo para esta bobina. Teniendo en cuenta que $\phi=(C t e) \cdot P$, la permeancia de las trayectorias de flujo del polo $\mathrm{N}$ se considerará positiva y la permeancia de las trayectorias de flujo del polo S se considerará negativa.

Tomando el eje del diente polo $\mathrm{N}$ de la bobina 1, (fase A), como eje de referencia, (Figura 5), la variación de la permeancia de las trayectorias de flujo del polo N, para esta bobina, será:

$$
P_{N}=P_{0}+P_{m} \cos \theta
$$

Análogamente, la variación de la permeancia de las trayectorias del polo $S$ de la bobina 1 es, $\left(Y_{e}=N_{r} \pi / 6, N_{r}\right.$ es el número de dientes y ranuras del rotor):

$$
-P_{S}=-\left[P_{0}+P_{m} \cos \left(\theta-N_{r} \frac{\pi}{6}\right)\right]
$$

Por lo tanto, la variación de la permeancia total, de las trayectorias de flujo de la bobina 1 de la fase A es igual a la suma de las expresiones (2) y (3).

$$
P_{1}=P_{m}\left[\cos \theta-\cos \left(\theta-N_{r} \frac{\pi}{6}\right)\right]
$$

La otra bobina de la fase A es la número 4. (Figura 5). La variación de la permeancia total de las trayectorias de flujo, para la bobina 4, es:

$$
P_{4}(\theta)=P_{m}\left[-\cos \left(\theta-N_{r} \pi\right)+\cos \left(\theta-N_{r} \frac{7 \pi}{6}\right)\right]
$$

Si las dos bobinas de cada fase se conectan en serie de tal forma que las tensiones generadas en ambas se sumen, deberá tenerse en cuenta si el número de dientes del rotor es par o impar. Si $\mathrm{N}_{\mathrm{r}}$ es par, la variación de permeancia total para la fase $A$ será $P_{1}-P_{4}$. Si $N_{r}$ es impar, la variación de permeancia total será $P_{1}+P_{4}$. Se recogen en la Tabla 1 los valores de las permeancias para cada una de las fases, en ambos casos. 


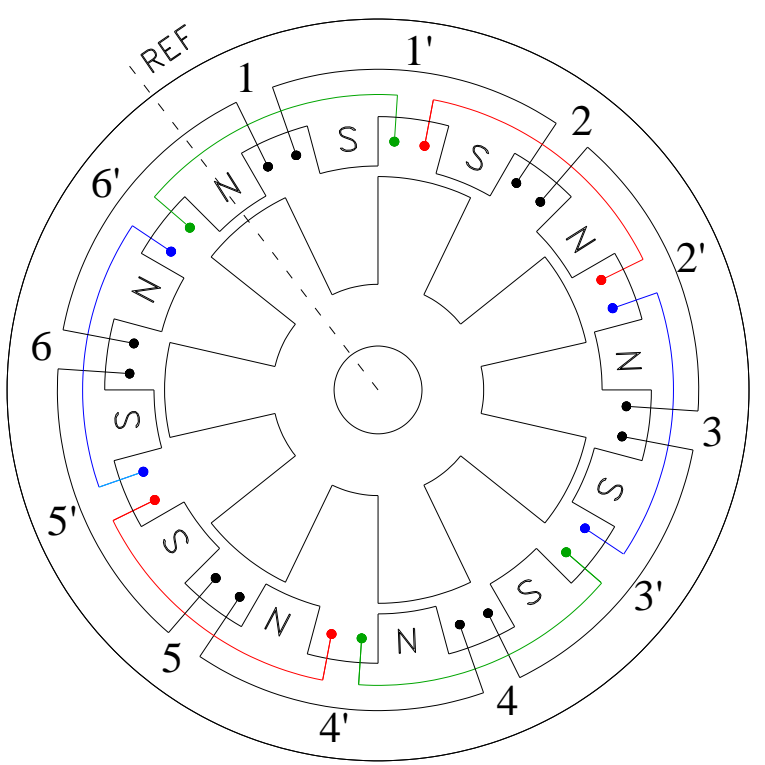

Fig. 5: Configuración de la sección del generador con 7 dientes en el rotor.

Tabla 1: Permeancias de las distintas fases.

\begin{tabular}{|l}
$N_{\mathrm{r}}$ Par: \\
\hline$P_{A}=P_{m}\left[\cos \theta-\cos \left(\theta-N_{r} \frac{\pi}{6}\right)+\cos \left(\theta-N_{r} \pi\right)-\cos \left(\theta-N_{r} \frac{7 \pi}{6}\right)\right]$ \\
$P_{B}=P_{m}\left[\cos \left(\theta-N_{r} \frac{4 \pi}{6}\right)-\cos \left(\theta-N_{r} \frac{5 \pi}{6}\right)+\cos \left(\theta-N_{r} \frac{10 \pi}{6}\right)-\cos \left(\theta-N_{r} \frac{11 \pi}{6}\right)\right]$ \\
$P_{C}=P_{m}\left[\cos \left(\theta-N_{r} \frac{8 \pi}{6}\right)-\cos \left(\theta-N_{r} \frac{9 \pi}{6}\right)+\cos \left(\theta-N_{r} \frac{2 \pi}{6}\right)-\cos \left(\theta-N_{r} \frac{3 \pi}{6}\right)\right]$ \\
$P_{A}=P_{m}\left[\cos \theta-\cos \left(\theta-N_{r} \frac{\pi}{6}\right)-\cos \left(\theta-N_{r} \pi\right)+\cos \left(\theta-N_{r} \frac{7 \pi}{6}\right)\right]$ \\
$P_{B}=P_{m}\left[\cos \left(\theta-N_{r} \frac{4 \pi}{6}\right)-\cos \left(\theta-N_{r} \frac{5 \pi}{6}\right)-\cos \left(\theta-N_{r} \frac{10 \pi}{6}\right)+\cos \left(\theta-N_{r} \frac{11 \pi}{6}\right)\right]$ \\
$P_{C}=P_{m}\left[\cos \left(\theta-N_{r} \frac{8 \pi}{6}\right)-\cos \left(\theta-N_{r} \frac{9 \pi}{6}\right)-\cos \left(\theta-N_{r} \frac{2 \pi}{6}\right)+\cos \left(\theta-N_{r} \frac{3 \pi}{6}\right)\right]$ \\
\hline
\end{tabular}

Las condiciones para obtener el número óptimo de dientes y ranuras del rotor son:

1. El flujo positivo entrante al rotor desde los dientes que son polos $\mathrm{N}$ debe retornar como flujo negativo a través de los dientes que son polos S, para completar las trayectorias. Esto quiere decir que el flujo total que entra en el rotor cuando es sumado, después de considerar sus signos correspondientes, debe ser igual a cero en cada instante y no depender del ángulo $\theta$. De no ser así, el devanado de excitación se autoinduciría tensión alternante. Se expresa la condición anterior en términos de permeancia. Como todas las permeancias están en paralelo, su efecto combinado es aditivo.

2. Para determinar las condiciones de máxima permeancia, se derivan con respecto a la variable $\theta$ y se igualan a cero las expresiones de los valores de permeancia obtenidos para las fases A, B y C.

3. Para la generación de tensión trifásica equilibrada, cuando la fase A tenga el valor máximo de tensión, las fases $B$ y C deberán tener la mitad del valor máximo, con polaridad opuesta a la de la fase $A$.

Al aplicar las expresiones de las permeancias, se obtienen las ecuaciones para cada una de las condiciones:

Condición 1: La permeancia total de las trayectorias de flujo positivo, (suma de las permeancias de los 6 dientes de estator que son polos $\mathrm{N}$ ), resulta, después de simplificar: 


$$
P_{\text {pos }}=6 P_{0}+2 P_{m} \cos \left(\theta-N_{r} \frac{11 \pi}{12}\right)\left[\cos \left(N_{r} \frac{3 \pi}{12}\right)+\cos \left(N_{r} \frac{5 \pi}{12}\right)+\cos \left(N_{r} \frac{11 \pi}{12}\right)\right]
$$

Análogamente, la permeancia total de las trayectorias de flujo negativo, (suma de las permeancias de los 6 dientes de estator que son polos S), será, después de simplificar:

$$
P_{\text {neg }}=6 P_{0}-2 P_{m} \cos \left(\theta-N_{r} \frac{11 \pi}{12}\right)\left[\cos \left(N_{r} \frac{3 \pi}{12}\right)+\cos \left(N_{r} \frac{5 \pi}{12}\right)+\cos \left(N_{r} \frac{11 \pi}{12}\right)\right]
$$

Haciendo $P_{\text {pos }}-P_{\text {neg }}=0$ se obtiene:

$$
4 P_{m} \cos \left(\theta-N_{r} \frac{11 \pi}{12}\right)\left[\cos \left(N_{r} \frac{3 \pi}{12}\right)+\cos \left(N_{r} \frac{5 \pi}{12}\right)+\cos \left(N_{r} \frac{11 \pi}{12}\right)\right]=0
$$

La expresión (8) no depende de $\theta$ cuando $\mathrm{N}_{\mathrm{r}}=1,2,4,5,6,7,8,10,11,13,14$... Como consecuencia de la hipótesis: $Y_{r} \geq Y_{e}$, los valores de $N_{r}$ deben estar comprendidos entre: $1 \leq N_{r} \leq 12$. Por lo tanto, el flujo magnético, en el devanado de excitación, se mantiene constante y no depende de $\theta$ para los valores $\mathrm{N}_{\mathrm{r}}=1$, 2, 4, 5, 6, 7, 8, 10 y 11 . Esta condición no se cumple cuando $N_{r}=3,9 \circ 12$.

Condición 2: La condición de máxima permeancia significa una mayor amplitud en la onda de variación del flujo ligado a una bobina del inducido y, por lo tanto, un mayor valor eficaz en la tensión inducida en circuito abierto. Para obtener el máximo valor de la permeancia de la fase $A$, para un valor fijado de $\mathrm{N}_{\mathrm{r}}$, deben derivarse las expresiones correspondientes, en la Tabla 1 , con respecto a la variable $\theta$ e igualarse a cero.

Al hacer $\mathrm{dP}_{\mathrm{A}} / \mathrm{d} \theta=0$, se obtiene:

$$
\begin{array}{ll}
\theta=\left(1+N_{r} \frac{7}{6}\right) \frac{\pi}{2} & \left(N_{r} \text { Par }\right) \\
\theta=N_{r} \frac{7 \pi}{12} & \left(N_{r} \text { Impar }\right)
\end{array}
$$

Si se sustituyen los valores de $\theta$ en las expresiones de las permeancias de la fase A, (Tabla 1), se obtienen:

$$
\begin{aligned}
& \left(P_{A \text { max }}\right)_{N_{r}=\text { Par }}=-4 P_{m} \cos \left(N_{r} \frac{\pi}{2}\right) \operatorname{sen}\left(N_{r} \frac{\pi}{12}\right) \\
& \left(P_{A \text { max }}\right)_{N_{r}=\text { Impar }}=-4 P_{m} \cos \left(N_{r} \frac{\pi}{2}\right) \operatorname{sen}\left(N_{r} \frac{\pi}{12}\right)
\end{aligned}
$$

El valor de $\mathrm{N}_{\mathrm{r}}$ debe ser elegido tal que el valor máximo de permeancia de la fase A sea lo más alto posible. Para ello, se derivan las expresiones (11) y (12) respecto de la variable $\mathrm{N}_{r}$ y se igualan a cero.

Se obtienen:

$$
\begin{array}{lll}
N_{r}=\frac{6}{7}(2 n \pm 1) & n=1,2,3 \ldots & \left(N_{r} \text { Par }\right) \\
N_{r}=1,71 \cdot n & n=1,2,3 \ldots & \left(N_{r} \text { Impar }\right)
\end{array}
$$

El único valor Par (entero) de $\mathrm{N}_{r}$ que cumple la condición (13) es $\mathrm{N}_{r}=6$, aunque, como veremos a continuación, no cumple la condición de tensión trifásica equilibrada. Ningún valor Impar (entero) de $\mathrm{N}_{\mathrm{r}}$ cumple la condición (14). La expresión del valor de la permeancia máxima para los valores enteros de $N_{r}=1,2,3, \ldots 12$, se obtiene sustituyendo el valor de $\mathrm{N}_{r}$ en las expresiones (11) y (12).

En todos los casos:

$$
P_{\text {A max }}=4 P_{m} \operatorname{sen}\left(N_{r} \frac{\pi}{12}\right)
$$

Los valores obtenidos se representan en la Figura 6. 


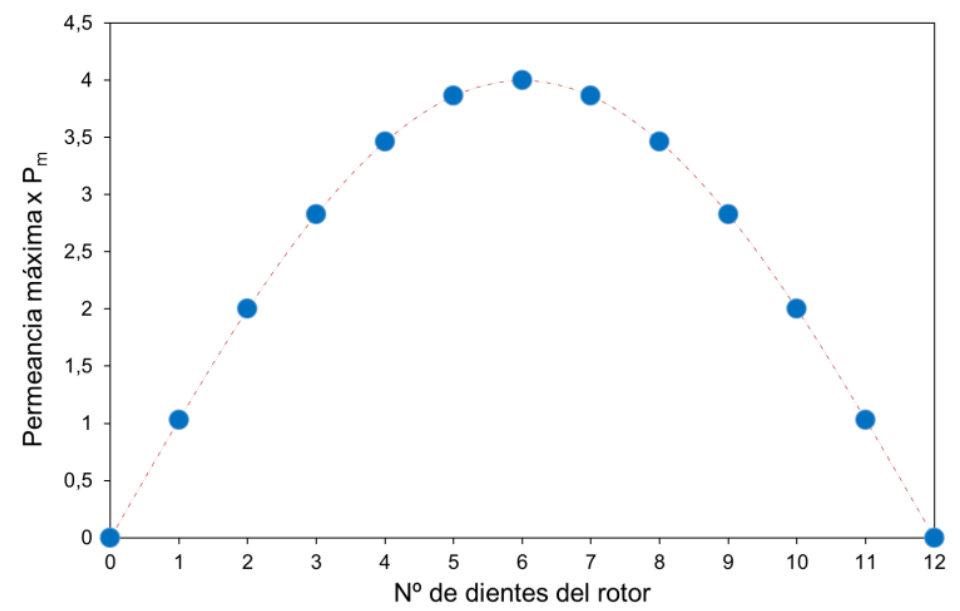

Fig. 6: Valor máximo de la permeancia de la fase A para cada valor de $\mathrm{N}_{\mathrm{r}}$.

Condición 3: Si se considera esta condición en términos de permeancias, cuando la permeancia de la fase A tenga el valor máximo, las fases $B$ y $C$ deberán tener la mitad del máximo valor de permeancia, con polaridad opuesta a la de la fase A. Para comprobar esta condición, se sustituyen los valores de $\theta$, obtenidos en las ecuaciones (9) y (10), en las expresiones de las permeancias de las distintas fases, recogidas en la Tabla 1, para obtener los valores de $\left(P_{B}\right)$ par y $\left(P_{C}\right)$ par $0\left(P_{B}\right)$ impar $y\left(P_{C}\right)$ impar, en el instante de tiempo en el que la fase $A$ pasa por el valor máximo PA máx. La condición a cumplir es, por tanto:

$\frac{1}{2} P_{A \max }=-P_{B}=-P_{C}$

En el caso de que $\mathrm{N}_{r}$ sea un número Par, la condición (16) se reduce a:

$$
\frac{1}{2}=-\cos N_{r} \frac{\pi}{3}=-\cos N_{r} \frac{2 \pi}{3}
$$

Si $\mathrm{Nr}$ es un número Impar, entonces:

$$
-\frac{1}{2}=-\cos _{r} \frac{\pi}{3}=\cos _{r} \frac{2 \pi}{3}
$$

La condición de tensión trifásica equilibrada se cumple para los valores: $N_{r}=1,2,4,5,7,8,10$ y 11 . Para elegir el valor óptimo del número de dientes y ranuras del Alternador Inductor, como generador trifásico de tensión sinusoidal equilibrada, deberán satisfacerse las ecuaciones (8), (15) y (16). Se descartan los valores de $\mathrm{N}_{\mathrm{r}}=3,6,9,12$, ya que no cumplen alguna de las condiciones. La condición de máxima permeancia, representada en la Figura 6, establece que el número más adecuado de dientes y ranuras del rotor es 507. (Cualquiera de ellos). Puesto que el Alternador Inductor es una máquina síncrona, existe una relación entre la velocidad de giro, ( $n, r p m)$, el número de dientes del rotor, $\left(\mathrm{N}_{\mathrm{r}}\right)$ y la frecuencia de la tensión inducida, (f), que viene dada por $f=N_{r} \cdot n / 60$.

\section{SIMULACIÓN}

Se ha aplicado un programa de elementos finitos, (FEMM), (Meeker, 2019), para comprobar la veracidad de los resultados del análisis. En la Figura 7 se representa la configuración de la sección del generador, con las líneas de flujo y los valores del campo magnético (B), cuando el número de dientes del rotor es $\mathrm{N}_{\mathrm{r}}=7$ y la corriente de excitación es le $=5 \mathrm{~A}$.

Se dispone de un equipo Feedback Electrical Machines Tutor EMT 180, utilizado para aplicaciones docentes (Feedback Instruments). Se configura un núcleo del estator y se construye un rotor de material magnético sinterizado, (soft magnetic composite), con el fin de obtener resultados experimentales que validen la simulación realizada con FEMM. Las bobinas de excitación, (6 bobinas), constan, cada una de ellas de $\mathrm{Ne}_{\mathrm{e}}$ $=175$ espiras. Las bobinas del inducido, ( 3 fases y 2 bobinas por fase), constan de $N_{i}=175$ espiras, cada una. El material magnético del estator es Ferrosil 216, (la curva de magnetización está definida en el manual del equipo), y el material del rotor es Somaloy 500 (Höganäs $A B$ ). 

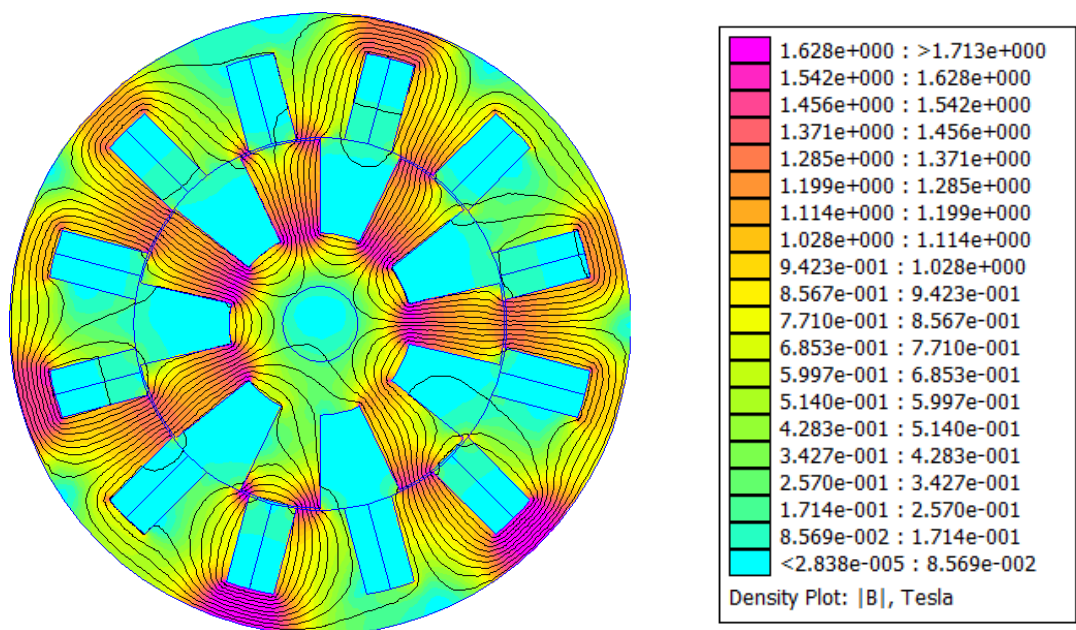

Fig. 7. Sección del generador, con $\mathrm{N}_{\mathrm{r}}=7$ e $\mathrm{l}_{\mathrm{e}}=5 \mathrm{~A}$, en la posición considerada como inicial ( $0^{\circ}$ eléctricos). Líneas de flujo magnético y valores de $\mathrm{B}$.

Las dimensiones de la sección del generador son: Radio del rotor: 50,4 mm; radio interior del estator: $51 \mathrm{~mm}$; radio exterior del estator: $85 \mathrm{~mm}$; profundidad de las ranuras del estator: $24 \mathrm{~mm}$; profundidad de las ranuras del rotor: 25,4 mm; radio del eje del rotor: 10,4 mm; longitud axial: $34 \mathrm{~mm}$. El material del eje del rotor es acero de baja permeabilidad, $\left(\mu_{\mathrm{r}}=300\right)$. Se hace variar la corriente en el devanado de excitación entre los valores $l_{e}=1 \mathrm{~A}$ hasta $l_{e}=10 \mathrm{~A}$. (Corriente continua). El inducido está en circuito abierto.

Se han obtenido, (para $1 \leq \mathrm{N}_{r} \leq 12$ ), los valores del flujo que atraviesa la bobina del inducido que se encuentra situada a la derecha de la bobina de referencia, (bobina 2, Figura 5). El valor máximo del armónico principal, $\left(A_{1}\right)$, en la onda de flujo corresponde al caso de 5 dientes en el rotor, cualquiera que sea el valor de la corriente de excitación, $\left(0,703 \cdot 10^{-3} \mathrm{~Wb}\right.$ para le $=5 \mathrm{~A}$, por ejemplo). Le siguen las configuraciones de 7 dientes, $\left(0,581 \cdot 10^{-3} \mathrm{~Wb}\right), 4$ dientes, $\left(0,522 \cdot 10^{-3} \mathrm{~Wb}\right)$, y 8 dientes, $\left(0,382 \cdot 10^{-3} \mathrm{~Wb}\right)$. En las demás configuraciones la amplitud de la onda es mucho más pequeña. Se indican, en la Figura 8 , los valores obtenidos para los casos de mayor interés, (4, 5, 7 y 8 dientes en el rotor). Se observa que, como consecuencia de la saturación magnética, el incremento de la amplitud de la onda de flujo, al aumentar el valor de la corriente de excitación, y, por lo tanto, el incremento de la tensión inducida en circuito abierto es cada vez menor a partir de le $\sim 5 \mathrm{~A}$.
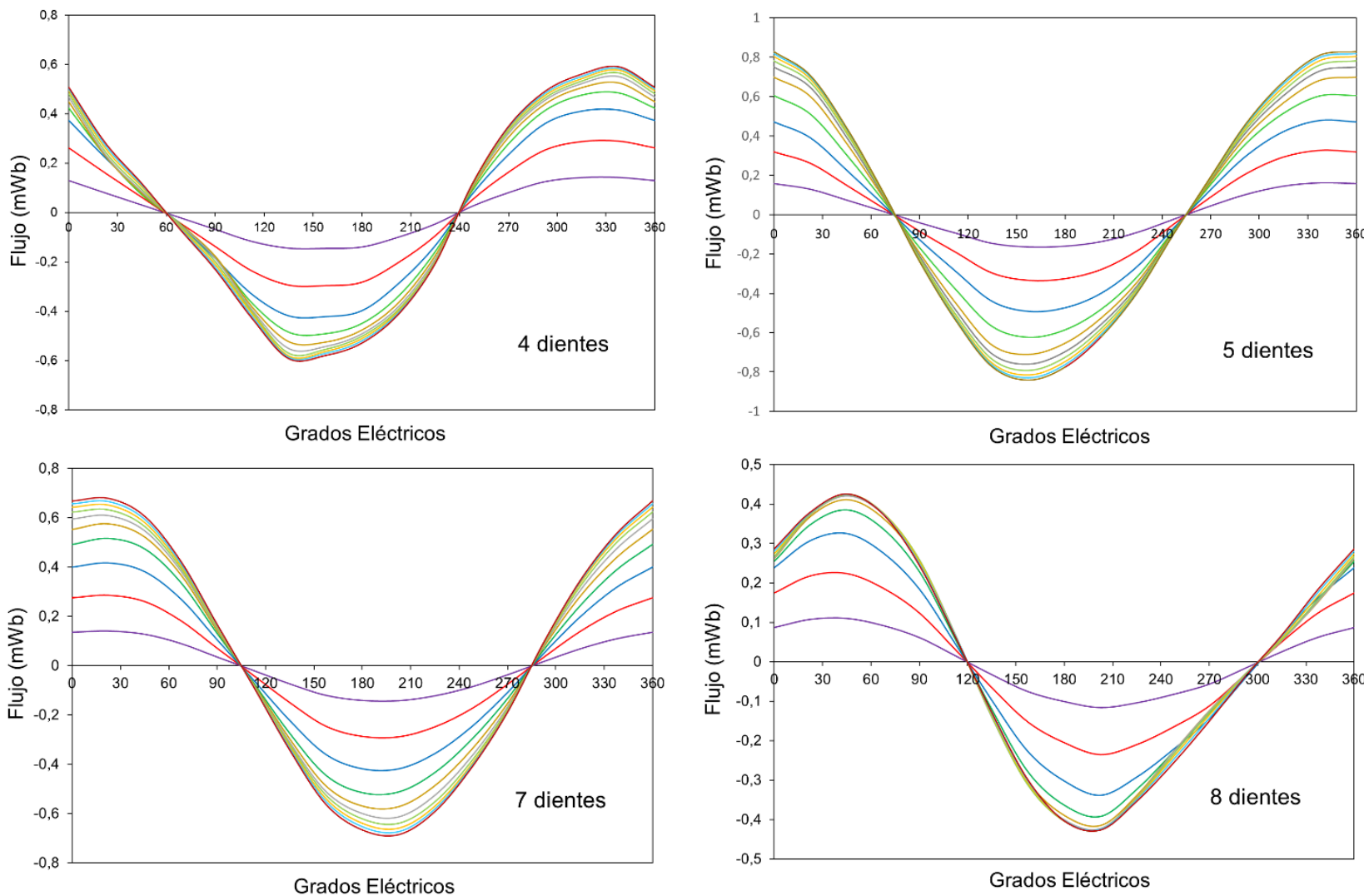

Fig. 8: Variación del flujo magnético en una bobina del inducido, para las configuraciones $N_{r}=4,5,7$ y 8 dientes. Valores de $\mathrm{l}_{\mathrm{e}}: 1 \mathrm{~A}-10 \mathrm{~A}$. 
Para determinar cuál de estas ondas es más aproximada a una función sinusoidal, se ha obtenido la Transformada Discreta de Fourier de cada una de las series de datos recogidos en la simulación mediante FEMM. Se toman 16 datos en cada una de las series, (intervalos de 22,5 grados eléctricos), lo que permite obtener el valor de los 8 primeros armónicos. En la Tabla 2 se indican los valores obtenidos para le $=5 \mathrm{~A}$. Aunque los porcentajes de los armónicos varían según sea el valor de le, se ha comprobado que el armónico más significativo es $A_{2}$, para todos los valores de $N_{r}$ y de $l_{e}$. La tensión inducida en cada bobina tendrá la misma forma de variación que la onda de flujo, ya que $e_{i}=N_{i}\left(d \phi_{i} / d t\right)$.

Tabla 2: Porcentajes del contenido en armónicos de las ondas de flujo para $l_{e}=5 \mathrm{~A}$.

\begin{tabular}{|c|cccc|}
\hline Armónico & $\mathrm{N}_{\mathrm{r}}=4$ & $\mathrm{~N}_{\mathrm{r}}=5$ & $\mathrm{~N}_{\mathrm{r}}=7$ & $\mathrm{~N}_{\mathrm{r}}=8$ \\
\hline $\mathrm{A}_{2}$ & $8,98 \%$ & $4,81 \%$ & $2,61 \%$ & $19,7 \%$ \\
$\mathrm{~A}_{3}$ & $2,95 \%$ & $0,13 \%$ & $0,01 \%$ & $1,90 \%$ \\
$\mathrm{~A}_{4}$ & $6,01 \%$ & $1,79 \%$ & $1,21 \%$ & $0,39 \%$ \\
$\mathrm{~A}_{5}$ & $0,58 \%$ & $0,80 \%$ & $0,41 \%$ & $1,14 \%$ \\
$\mathrm{~A}_{6}$ & $0,67 \%$ & $0,16 \%$ & $1,05 \%$ & $0,32 \%$ \\
$\mathrm{~A}_{7}$ & $1,39 \%$ & $0,06 \%$ & $0,07 \%$ & $0,73 \%$ \\
$\mathrm{~A}_{8}$ & $0,32 \%$ & $1,07 \%$ & $0,19 \%$ & $0,60 \%$ \\
\hline
\end{tabular}

Se ha obtenido el coeficiente de distorsión (k) en cada caso. Los resultados se recogen en la Figura 9.

$$
k(\%)=\frac{\sqrt{\sum_{i \neq 1} U_{i}^{2}}}{U_{1}} \cdot 100
$$

En esta ecuación, $U_{i}$ es el valor eficaz de la amplitud del armónico i. En los alternadores convencionales es recomendable: $\mathrm{k}<5 \%$ para $\mathrm{S}>300 \mathrm{kVA}, \mathrm{y} \mathrm{k}<10 \%$ para $1 \mathrm{kVA}<\mathrm{S}<100 \mathrm{kVA}$.

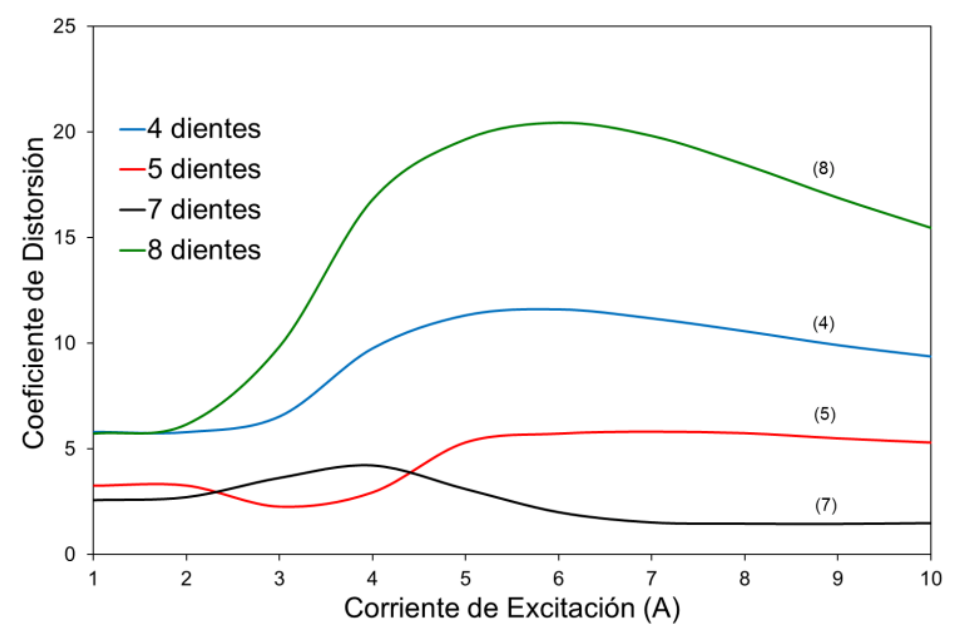

Fig. 9: Variación del coeficiente de distorsión en las ondas del flujo ligado a una bobina del inducido, para $\mathrm{N}_{\mathrm{r}}=4,5,7$ y 8 dientes.

La conclusión del análisis del circuito magnético es que las configuraciones de 5 y de 7 dientes de rotor deberían proporcionar las mismas formas de onda de variación de flujo, ya que el flujo es igual a la permeancia por una constante. Igualmente, las ondas de flujo de las configuraciones de 4 y 8 dientes deberían ser iguales. Las diferencias encontradas al aplicar la simulación se deben a que la variación de la permeancia en un diente del estator, (en un paso polar $\mathrm{Y}_{\mathrm{e}}$ ), como consecuencia del giro del rotor, no es sinusoidal y además depende del número de dientes del rotor.

En la Figura 10 se representan las formas de onda de la variación del flujo en un diente cualquiera del estator, al girar el rotor, para cada una de las configuraciones de interés: 4, 5, 7 y 8 dientes de rotor. La corriente de excitación varía entre los valores $l_{e}=1 \mathrm{~A}$ hasta $l_{e}=10 \mathrm{~A}$. Estas formas de onda son iguales para todos los dientes del estator, aunque el desfase dependerá del diente considerado. Se han representado las formas de onda correspondientes al diente número 3. (Diente $\mathrm{N}$ de la bobina 3, en la Figura 5). Se han obtenido los coeficientes de distorsión ( $k$ ) de las ondas de flujo representadas en la Figura 10. El resultado se indica en la Figura 11. 

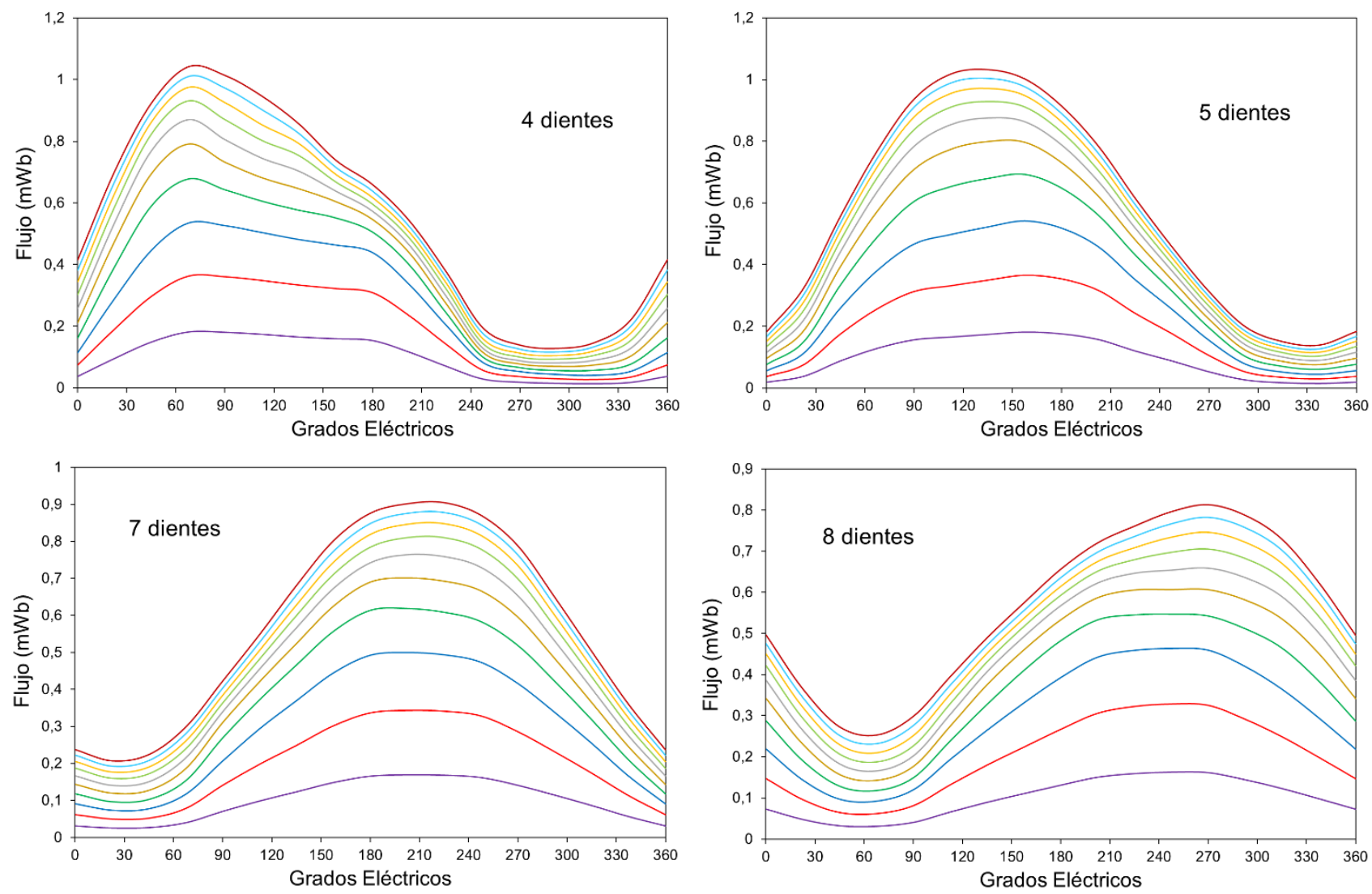

Fig. 10: Variación del flujo magnético en un diente del estator, para las configuraciones $\mathrm{N}_{\mathrm{r}}=4,5,7$ y 8 dientes. Valores de le: $1 \mathrm{~A}-10 \mathrm{~A}$.

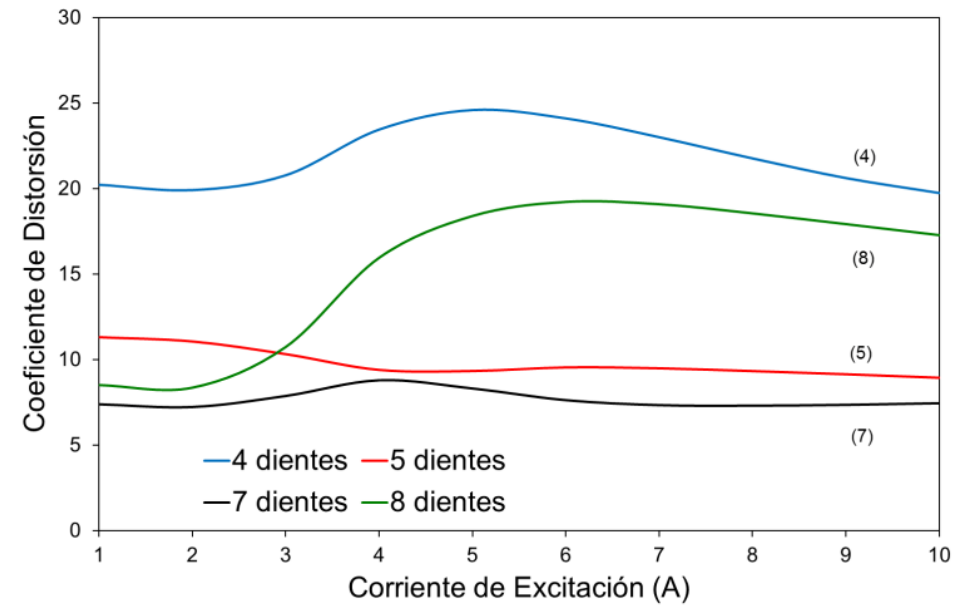

Fig. 11: Variación del coeficiente de distorsión en las ondas del flujo en un diente del estator, para las configuraciones $\mathrm{N}_{\mathrm{r}}=4,5,7$ y 8 dientes.

Para obtener el valor de la tensión inducida, en una bobina del inducido, se aplicará la expresión:

$$
\emptyset_{i}(\theta)=\emptyset_{i \max } \cos \left(\theta_{e}+\alpha\right)
$$

Aunque la forma de onda de la variación del flujo en un diente del estator no es sinusoidal, se pone de manifiesto, con los resultados obtenidos en la simulación, que la onda de variación del flujo en cada una de las bobinas del inducido lo es con bastante aproximación. Los valores del flujo, en una bobina del inducido, obtenidos en la simulación y representados en la figura 8 , son, en cada caso, comparables con las funciones: 4 dientes: $A_{1} \cos (\theta+301)$; 5 dientes: $A_{1} \cos (\theta+151) ; 7$ dientes: $A_{1} \cos (\theta-151)$; y 8 dientes: $A_{1} \cos (\theta-$ 301). $A_{1}$ es el valor correspondiente a la amplitud del armónico fundamental, obtenido para cada una de las ondas de flujo representadas en la Figura 8. Para obtener la tensión inducida en una bobina de fase, son aplicables las expresiones: 


$$
\begin{aligned}
& e_{i}=-\frac{d \psi_{i}}{d t} \\
& \psi_{i}=N_{i} \cdot \phi_{i}=N_{i} \phi_{i \max } \cos \left(\theta_{e}+\alpha\right) \\
& \theta_{e}=2 \pi f \cdot t \quad y \quad \theta=\Omega \cdot t \quad \quad\left(\theta_{e}=\text { grados eléctricos y } \theta=\right.\text { grados mecánicos) } \\
& \theta_{e}=N_{r} \cdot \theta \quad \text { (Relación de sincronismo) } \\
& f=\frac{N_{r} \cdot \Omega}{2 \pi} \quad \begin{array}{l}
\psi_{i}(\theta)=N_{i} \phi_{i \max } \cos (2 \pi f t+\alpha) \\
e_{i \max }=2 \pi f N_{i} \phi_{i \max }
\end{array}
\end{aligned}
$$

El valor eficaz de la tensión inducida es:

$$
E_{\text {eficaz }}=\frac{e_{i \max }}{\sqrt{2}}
$$

Cada fase tiene dos bobinas conectadas en serie; por lo tanto, la tensión de fase será: $V_{\text {fase }}=2 \mathrm{E}_{\text {eficaz }}$ y la tensión de línea de salida será: Ulínea $=\sqrt{ } 3 \cdot V_{\text {fase }}$. Teniendo en cuenta que $\mathrm{N}_{\mathrm{i}}=175$ espiras, si se considera una velocidad de giro del alternador: $n=2.000 \mathrm{rpm}$, se obtienen los valores, para Ulínea, representados en la Figura 12.

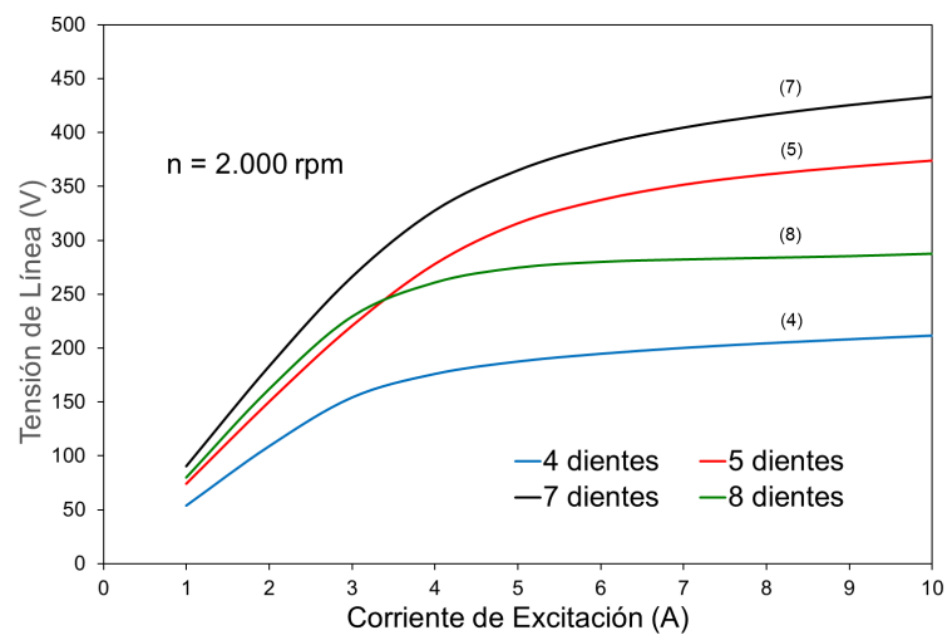

Fig. 12: Valores de la tensión de salida en circuito abierto, ( $\left.\bigcup_{\text {línea }}\right)$, para $n=2.000 \mathrm{rpm}$.

\section{ANÁLISIS EXPERIMENTAL}

Para comprobar la validez de la simulación se comparan los resultados que se han obtenido, al aplicar el programa FEMM, con los que se obtienen de forma experimental al utilizar un prototipo real. La configuración de la máquina se representa en la Figura 13.

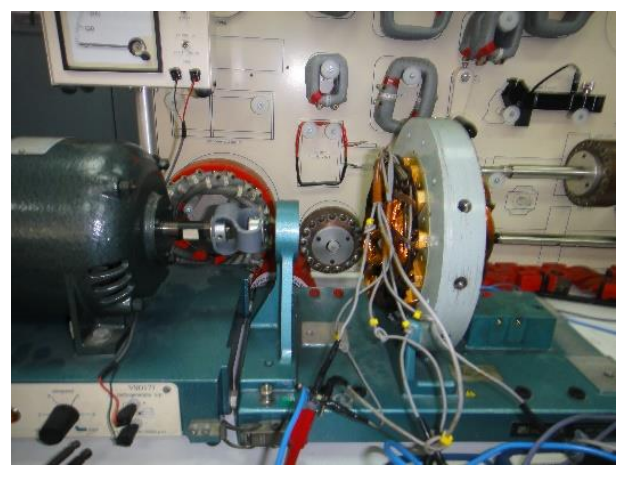

Fig. 13: Configuración del generador, con el motor de arrastre incorporado. 
Se utiliza el núcleo del estator del equipo Feedback, al que se le han añadido las bobinas de excitación y del inducido (175 espiras en cada bobina). (Figura 14a). El rotor se ha construido del material denominado Somaloy 500. (Figura 14b). El Somaloy 500 es un aglomerado sinterizado de materiales magnéticos blandos, (soft magnetic composite, SMC) y presenta menores pérdidas magnéticas que la chapa magnética convencional. Se han mecanizado y pegado 2 piezas de $17 \mathrm{~mm}$ de ancho y se ha añadido un elemento circular para la fijación al eje de giro.
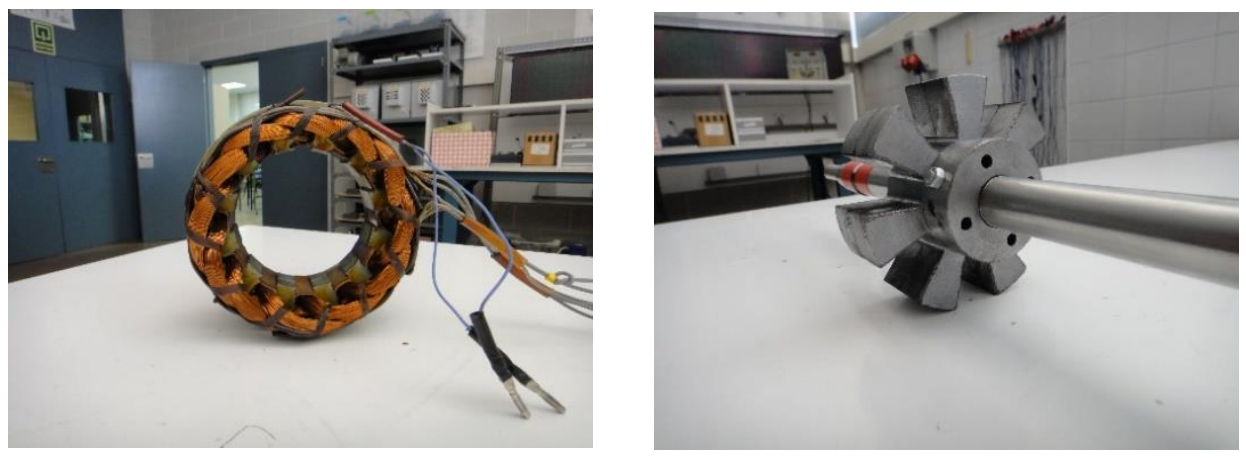

Fig. 14: a) Estator. b) Rotor de SMC con 7 dientes.

Limitaciones en la potencia y la velocidad de rotación del motor de arrastre disponible obligan a realizar los ensayos con corriente de excitación reducida y velocidades inferiores a $3000 \mathrm{rpm}$. Se representan, en la Figura 15a, las formas de onda de las tensiones de salida de fase y de línea, en circuito abierto, para le $=2 \mathrm{~A}$ y $\mathrm{n}=2.000 \mathrm{rpm}$. Los valores eficaces son $\mathrm{V}_{\text {fase }}=102 \mathrm{~V}$ y Ulínea $=175 \mathrm{~V}$. La frecuencia de la tensión de salida es igual a $233,3 \mathrm{~Hz}$. Al conectar, en estrella, las tres fases del inducido, se obtienen valores algo diferentes en los porcentajes de los armónicos, respecto de los que aparecen en las tensiones de fase. Estos coinciden, con buena aproximación, con los obtenidos en la simulación para las ondas del flujo ligado a una bobina del inducido. La distribución de los armónicos de la tensión de línea es más uniforme, destacando ligeramente los armónicos $A_{5}$ y $A_{11}$. (Figura 15b). El coeficiente de distorsión de la onda es $k=4,25 \%$.

En la simulación con FEMM se han obtenido, (para $l_{e}=2 \mathrm{~A}$ y $n=2.000 \mathrm{rpm}$ ), los valores eficaces: $\mathrm{V}_{\text {fase }}=$ $105,69 \mathrm{~V}$, Ulínea $=183,07 \mathrm{~V}$. El coeficiente de distorsión de la onda de flujo, para $\mathrm{l}_{\mathrm{e}}=2 \mathrm{~A}$, es $\mathrm{k}=2,72 \%$. Teniendo en cuenta las tolerancias en las dimensiones reales de la máquina, estas diferencias son pequeñas. En la Figura 16 se representa la variación de la tensión de salida, (Ulínea), en función de la velocidad de rotación, en circuito abierto.
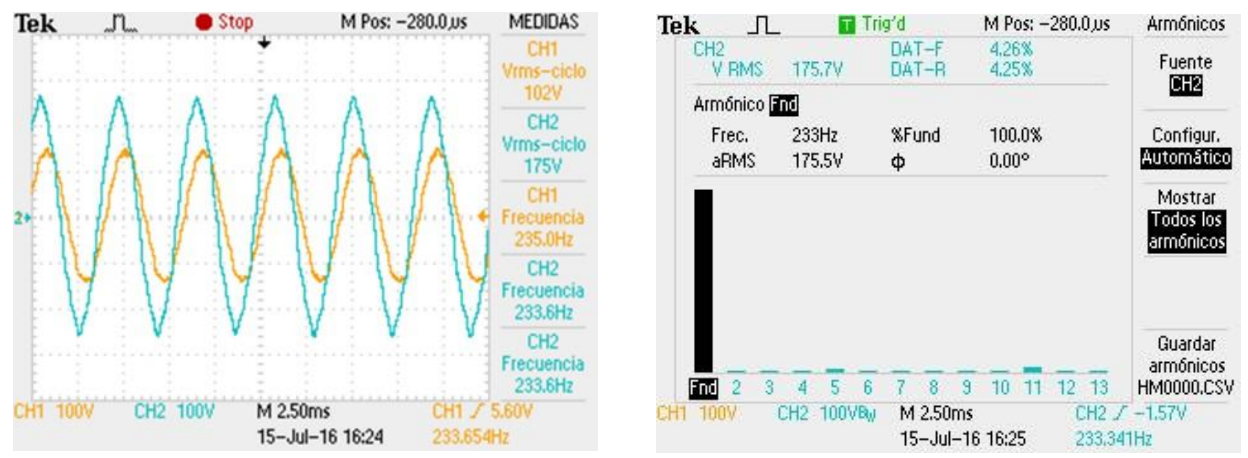

Fig. 15: a) Formas de onda de las tensiones de salida de fase, (naranja), y de línea, (verde), en circuito abierto. b) Análisis de armónicos de la tensión de línea y valor del coeficiente de distorsión.

Se han obtenido experimentalmente los parámetros y las prestaciones del alternador, en estado estacionario. El análisis, por su extensión, deberá ser objeto de un trabajo posterior. En la Figura 17 se representan las características externas, para carga resistiva. ( $\mathrm{l}_{\mathrm{e}}=2 \mathrm{~A}$ y velocidades de rotación de $1.000,1.500$ y $2.000 \mathrm{rpm}$ ). La potencia de salida máxima, a $2.000 \mathrm{rpm}$, es igual a $125 \mathrm{~W}$ y el rendimiento máximo es igual a 0,42.

Estos valores no son significativos porque han sido obtenidos en condiciones de excitación muy inferiores a las óptimas, debido a las limitaciones ya comentadas del motor de mando. El control de la tensión de salida puede hacerse de forma similar al de los alternadores convencionales. El diseño de un prototipo para aplicaciones industriales deberá realizarse atendiendo a criterios geométricos y electromagnéticos más adecuados, (relación óptima entre la longitud axial y el diámetro del rotor según la potencia requerida, valores del campo magnético en el entrehierro del orden de 1,5 T y densidades de corriente del orden de $5 \mathrm{~A} / \mathrm{mm}^{2}$ ). 


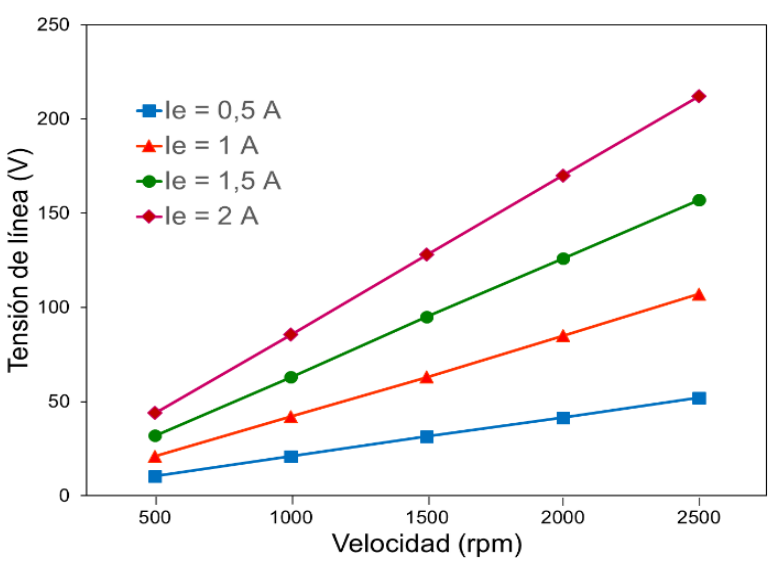

Fig. 16: Variación de la tensión de salida en circuito abierto, (Ulínea), en función de la velocidad de rotación.

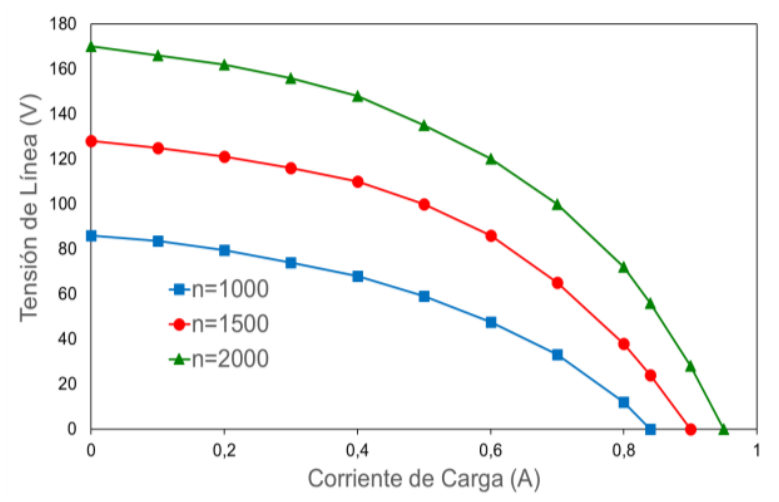

Fig. 17: Características externas para carga resistiva.

\section{DISCUSIÓN FINAL}

Se configura un alternador inductor heteropolar trifásico, con excitación mediante un devanado de corriente continua, con una disposición en la ubicación de las bobinas de excitación y de inducido tal que el flujo magnético ligado a cada una de las bobinas del inducido cambia de sentido, lo que aumenta el valor de la tensión inducida, para unos valores dados en la corriente de excitación, si se lo compara con una configuración de flujo pulsante. Partiendo de una configuración del estator determinada, se trata de elegir el número de dientes del rotor más adecuado para que la tensión de salida, en circuito abierto, sea óptima, en valor eficaz y menor contenido en armónicos. El análisis teórico determina que 5 o 7 dientes en el rotor son los valores más adecuados. La simulación establece que un rotor de 7 dientes presenta un coeficiente de distorsión más bajo que el de 5 dientes, aunque el valor máximo de la onda de flujo en una bobina del inducido es ligeramente menor. Se ha construido un prototipo utilizando el núcleo del estator de un equipo para aplicaciones docentes y se han mecanizado y pegado dos piezas del material magnético denominado Somaloy 500 para configurar un rotor con 7 dientes. Los resultados experimentales obtenidos, en el comportamiento estacionario, coinciden con una buena aproximación con los previstos en la simulación.

Las posibles aplicaciones del alternador son variadas y cubren todo el rango de velocidades: A bajas velocidades, $(<3.000 \mathrm{rpm})$, conectado a un aerogenerador eólico; a velocidades medias, $(3.000-20.000$ rpm), en plataformas generadoras móviles y en vehículos híbridos; y en altas velocidades, (20.000 - 100.000 $\mathrm{rpm}$ ), acoplado al eje de una turbina de gas. No necesita un sistema de engranajes y su bajo coste y simplicidad lo hacen interesante para algunas aplicaciones (Martínez y Andrada, 2017), ya que presenta también otras ventajas, como alta densidad de potencia, gran rigidez mecánica, bajo nivel de ruido, reducción del peso y volumen del conjunto y ausencia de mantenimiento. Por todo ello, el alternador inductor heteropolar con inversión de flujo, en sus diferentes formas de excitación, encuentra cada día un mayor interés en la industria, para la generación de energía eléctrica.

\section{CONCLUSIONES}

De acuerdo con el trabajo presentado y los resultados obtenidos, se pueden plantear las siguientes conclusiones principales:

1. El análisis teórico basado en la obtención de las permeancias de las trayectorias del flujo, en el circuito magnético, determina que los valores de 5 o 7 dientes en el rotor son los más idóneos, obteniéndose en 
ambos casos la misma forma de onda de variación de permeancia y, por lo tanto, de variación del flujo magnético y de la tensión inducida en circuito abierto. Como consecuencia de la hipótesis de variación sinusoidal de la permeancia, la tensión inducida no contiene armónicos.

2. La simulación mediante FEMM proporciona los valores más altos del flujo magnético ligado a una bobina del inducido para la configuración de 5 dientes, seguida de la configuración de 7 dientes. El coeficiente de distorsión de la onda de flujo es menor en la configuración de 7 dientes que en la de 5 dientes. Para una velocidad de giro determinada, el mayor valor eficaz de la tensión de salida, en circuito abierto, ocurre para la configuración de 7 dientes debido a que la frecuencia de la tensión generada es mayor.

3. Se ha construido un prototipo con 7 dientes en el rotor y los resultados experimentales obtenidos, en el comportamiento estacionario, coinciden con una buena aproximación con los previstos en la simulación.

\section{REFERENCIAS}

Akuru, U. B. y Kamper, M. J., Formulation and Multiobjective Design Optimization of Wound-Field Flux Switching Machines for Wind Energy Drives, doi: 10.1109/TIE.2017.2721928, IEEE Trans on Industrial Electronics, 65 (2), 1828 - 1836 (2018)

Das Gupta, A. K., Mathematical Analysis of Inductor Alternators, doi: 10.1109/AIEEPAS.1960.4500836, AIEE Trans. Part III: Power, Apparatus and Systems, 79 (3), 684 - 689 (1960)

Feedback Instruments Limited., Crowborough (United Kingdom), en la web: http://www.feedback-instruments.com/ products/. Último acceso: 12 de Agosto (2019)

Gaussens, B., Hoang, E., Lécrivain, M. y Manfe, P., A Hybrid-Excited Flux-Switching Machine for High-Speed DCAlternator Applications, doi:10.1109/TIE.2013.2281152, IEEE Trans on Industrial Electronics, 61 (6), 2976 - 2989 (2014)

Höganäs, AB, Högänas (Sweden), en la web: https://www.hoganas.com/somaloy/. Último acceso: 12 de Agosto (2019)

Kerala Electrical \& Allied Engineering Company Ltd., Kundara (India), en la web: http://kel.co.in/products/. acceso: 12 de Ago. (2019)

Lamme, B. G., Data and Tests on a 10.000 cycle per second Alternator, doi: 10.1109/T-AIEE.1904.4764469, AIEE Trans, 23 (1), 417 - 428 (1904)

Li, Y., Bobba, D. y Sarlioglu, B., A Novel 6/4 Flux-Switching Permanent Magnet Machine Designed for High-Speed Operations, doi:10.1109/TMAG.2016.2547366, IEEE Trans on Magnetics, 52 (8), Art № 8107109 (2016)

Marchbanks, M. J., Coreless Induction Furnaces, doi: 10.1049/ji-2.1946.0111, Journal IEE. Part II: Power Engineering, 93 (36), 517 - 529 (1946)

Martínez, F. y Andrada, P., Generadores Síncronos de Alta Velocidad. Una Evaluación Crítica, doi: 10.4067/S071807642017000200006, Información Tecnológica, 28 (2), 47 - 58 (2017)

Meeker, D., Finite Element Method Magnetics (FEMM), en la web: http://www.femm.info/ wiki/HomePage. Último acceso: 12 de Agosto (2019)

Milspec Manufacturing Pty Limited, Albury (Australia), en la web: http://milspecmanufacturing.com/products/alternators/ 360-ac/. Último acceso: 12 de Agosto (2019)

Rauch, S. E. y Johnson, L. J., Design Principles of Flux Switch Alternators, doi: 10.1109/AIEEPAS.1955.4499226, IEE Trans. Part III: Power Apparatus and Systems, 74 (3), 1261 - 1268 (1955)

Severson, E., Nilssen, R. y Undeland, T., Magnetic Equivalent Circuit Modeling of the AC Homopolar Machine for Flywheel Energy Storage, doi: 10.1109/TEC.2015.2441040, IEEE Trans on Energy Conversion, 30 (4), 1670 - 1678 (2015)

Shen, J. X. y Fei, W. Z., Permanent Magnet Flux Switching Machines. Topologies, Analysis and Optimization, doi: 10.1109/PowerEng.2013.6635633, 4th International Conference on Power Engineering, Energy and Electrical Drives, 352 - 366, Istanbul-Turquía, 13 - 17 de Mayo (2013)

Sivasubramaniam, K., Zhang T. y otros seis autores, Development of a High Speed HTS Generator for Airborne Applications, doi: 10.1109/TASC.2009.2017758, IEEE Trans on Applied Superconductivity, 19 (3), 1656 - 1661 (2009)

Walker, J. H., High Frequency Alternators, doi: 10.1049/ji-2.1946.0016, Journal IEE. Part II: Power Engineering, 93 (31), 67 - 80 (1946)

Walker, J. H., The Theory of the Inductor Alternator, doi: 10.1049/ji-2.1942.0037, Journal IEE. Part II: Power Engineering, 89 (9) 227 - 241 (1942)

Zhang, G., Hua, W., Cheng, M. y Liao J. Design and Comparison of Two Six-Phase Hybrid-Excited Flux-Switching Machines for EV/HEV Applications, doi: 10.1109/TIE.2015.2447501, IEEE Trans on Industrial Electronics, 63 (1), 481 - 493 (2016)

Zhu, Z. Q., Zhou, Y. J., Chen, J. T. y Gren, J. E., Investigation of Nonoverlapping Stator Wound-Field Synchronous Machines, doi: 10.1109/TEC.20152423280, IEEE Trans on Energy Conversion, 30 (4), 1420 - 1427 (2015) 